\title{
Niedriger BMI ist mit einem höheren Lymphopenierisiko assoziiert
}

Fragestellung: Hängen Lymphopenien unter Fingolimod vom Body-Mass-Index (BMI) ab, oder sind Patienten mit niedrigem BMI überdosiert?

Hintergrund: Ein wichtiger Sicherheitsaspekt bei Patienten, die mit Fingolimod behandelt werden, ist das mögliche Auftreten von signifikanten Lymphopenien, insbesondere das Unterschreiten der kritischen Grenze von 0,2 x $10^{9}$ Lymphozyten/l. Gemäß den allgemeinen Therapieempfehlungen muss beim Unterschreiten dieser Grenze die Behandlung mit Fingolimod ausgesetzt werden. Bisher liegen leider kaum Daten oder Strategien vor, die es erlauben abzuschätzen, inwieweit ein bestimmter Patient für die Entwicklung einer ausgeprägten Lymphopenie prädisponiert ist.

Obwohl die individuellen Unterschiede zwischen den verschiedenen Patienten bekannt sind, werden alle Patienten unabhängig von Gewicht, BMI und genetisch determinierten $\mathrm{Me}$ tabolisierungraten grundsätzlich mit der gleichen Dosierung behandelt, im Falle von Fingolimod mit 0,5 mg täglich. Diese Studie untersuchte die Frage,

Warnke C, Dehmel T, Ramanujam R et al. Initial lymphocyte count and low BMI may affect fingolimod-induced lymphopenia. Neurology 2014; 83: $2153-7$ ob eine niedrige initiale Lymphozytenzahl, ein niedriger BMI, das Alter oder das Geschlecht mit einem höheren Risiko einer ausgeprägten Lymphopenie assoziiert ist.
Patienten und Methodik: Im Rahmen einer einarmigen, offenen Multicenterstudie wurden Patienten, die mit Fingolimod 0,5 mg behandelt wurden, entsprechend gemonitort und im Anschluss die Daten im Rahmen einer schwedischen Pharmakovigilanzstudie validiert und bewertet.

Ergebnisse: In die Studie wurden 418 Patienten aus Deutschland und 438 Patienten aus Schweden eingeschlossen. Der kritische Nadir von 0,2 wurde bei $15 \%$ aller Patienten beobachtet. Patienten mit einen niedrigen Lymphozytenausgangswert und Patienten mit einem BMI unter 18,5 hatten ein signifikant erhöhtes Risiko, eine kritische Lymphopenie zu entwickeln (26\% der Patienten).

Von den Patienten, bei denen beide klinischen Aspekte vorlagen, wiesen immerhin $46 \%$ die kritische Lymphopenie auf. In der deutschen Kohorte zeigten sich keine Unterschiede hinsichtlich des Auftretens von Infektionen zwischen den Patienten mit ausgeprägter Lymphopenie versus Patienten mit geringer Lymphopenie beziehungsweise normaler Lymphozytenzahl.

Schlussfolgerungen: Die Autoren schließen aus den hier erhobenen Daten, dass Patienten mit niedriger Lymphozytenzahl zu Beginn der Behandlung und Patienten mit einem niedrigen BMI ein höheres Risiko haben, eine kritische Lymphopenie zu entwickeln und entsprechend aufmerksamer beobachtet werden sollten.

\section{- Kommentar von Volker Limmroth, Köln-Merheim}

\section{Völlig vernachlässigt: der BMI}

Eigentlich haben wir es immer gewusst und jeder Pharmakologe schüttelt sowieso mit dem Kopf, dass Patienten mit einem Gewicht von $45 \mathrm{~kg}$ die gleiche Dosis erhalten wie Patienten mit $150 \mathrm{~kg}$. Alle Dosierungen, die heute aufgrund von klinischen Studien durchgeführt werden, sind auf einen Durchschnittspatienten von $75 \mathrm{~kg}$ und einen BMI von 25 zugeschnitten. Damit stimmt die Dosierung wahrscheinlich auch für $80 \%$ aller Patienten. Bei Patienten mit einem niedrigen BMI müssen wir aber mit einem stärkeren Medikamenteneffekt als bei Patienten mit einem hohen BMI rechnen - keine Überraschung. Umgekehrt müssen wir uns gelegentlich sicher auch fragen, wenn ein Patient mit einem BMI von 45 nicht wirklich von der Medikation profitiert, ob dieser nicht möglicherweise auch unterdosiert sein könnte.

Die Studie ist für die tägliche Routine sehr hilfreich und man möchte eigentlich mehr Untersuchungen dieser Art sehen. Die wichtige Botschaft ist leicht nachvollziehbar: Patienten mit einem niedrigen BMI müssen besser beobachtet werden und haben erwartungsgemäß ein höheres Nebenwirkungsrisiko.
Interessant ist aber auch der Aspekt, dass Patienten mit dem als kritische Lymphopenie definierten Abfall unter 0,2 keine erhöhte Infektionsrate aufwiesen. Insofern stellt sich auch die Frage, inwieweit die Grenze von 0,2 klinisch sinnvoll beziehungsweise nützlich ist. Diese Frage sollte auch demnächst geklärt werden.

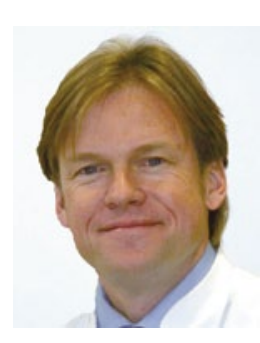

Prof. Dr. med. Volker Limmroth, Köln-Merheim

Chefarzt der Klinik für Neurologie und Palliativmedizin Köln-Merheim E-Mail: LimmrothV@kliniken-koeln.de 